\title{
Implementation of a loss-compensated recirculating delayed self-heterodyne interferometer for ultranarrow laser linewidth measurement
}

\author{
Xiaopei Chen, Ming Han, Yizheng Zhu, Bo Dong, and Anbo Wang
}

\begin{abstract}
Ultranarrow laser linewidth measurement using an optimized loss-compensated recirculating delayed self-heterodyne interferometer is described. An experimental setup is constructed to measure subkilohertz laser linewidths. The system parameters are optimized to obtain the best beat signals. The experimental results agree well with the theoretical analysis. Two methods of linewidth interpretation are presented and analyzed based on the experimental results. It is proved that a loss-compensated recirculating delayed self-heterodyne interferometer is an effective tool for measuring an ultranarrow laser linewidth. (C) 2006 Optical Society of America
\end{abstract}

OCIS codes: $140.3430,120.3180$.

\section{Introduction}

With the rapid development of rare-earth-doped fibers, fiber lasers with linewidths as narrow as the subkilohertz regime have been commercialized. ${ }^{1}$ The narrow linewidth and excellent frequency noise characteristics make ultranarrow linewidth lasers more attractive in a large variety of areas. First, ultranarrow linewidth lasers have extremely long coherence lengths. Fiberoptic sensing systems can take advantage of the long coherent lengths to realize long-distance coherent detection, which is widely used for security or military applications, oil or gas well surveying, earthquake detection and high-voltage power lines. ${ }^{2}$ Second, ultranarrow linewidth lasers have exceptionally low phase noise. This property greatly improves the sensitivity of interferometric fiber-optic sensors, such as fiber-optic hydrophone arrays for military sonar applications ${ }^{2}$ and in seismic surveying for the oil and gas industry. Therefore an accurate measurement of the laser linewidths of ultranarrow lasers becomes critically important.

The authors are with the Center for Photonics Technology, Bradley Department of Electrical and Computer Engineering, Virginia Polytechnic Institute and State University, Blacksburg, Virginia 24061-0111. X. Chen's e-mail address is xichen5@vt.edu.

Received 10 February 2006; accepted 15 May 2006; posted 6 June 2006 (Doc. ID 67973).

$0003-6935 / 06 / 297712-06 \$ 15.00 / 0$

(C) 2006 Optical Society of America
When the linewidth of a single-frequency laser is of the order of $1 \mathrm{kHz}$, typical grating-based optical spectrum analyzers (OSAs) and scanning filter methods are not able to offer the required measurement resolution. Therefore heterodyne detection, frequency discriminator, and self-heterodyne detection methods are proposed for high resolution linewidth measurement. ${ }^{3}$ But these methods have limitations when measuring the laser linewidth of the order of a subkilohertz. For heterodyne detection, linewidth measurement needs a more stable and even narrower linewidth laser as a reference. To measure and calibrate the reference laser is often an even more difficult task. In a conventional delayed self-heterodyne interferometer (DSHI), ultralong fiber delay is required instead of a reference laser. But for an ultranarrow linewidth laser the fiber delay could be impractically long. Though literally frequency discrimination methods can be used for any laser, including the ultranarrow linewidth lasers, they are found to be complex, expensive, and incomplete. The interpretation of the measurement results from frequency discriminators is especially difficult.

We use an improved delayed self-heterodyne detection in which a loss-compensated fiber loop is used as the delay line. This method is referred to as the losscompensated recirculating delayed self-heterodyne interferometer (LC-RDSHI) and was proposed by Dawson et al..$^{4}$ and Park et al..$^{5}$ in 1992. Han and Wang $^{6}$ did theoretical analysis of the LC-RDSHI in 2004. Here, we verified the results of LC-RDSHI in Ref. 6 by experiment and for what we believe to be the 


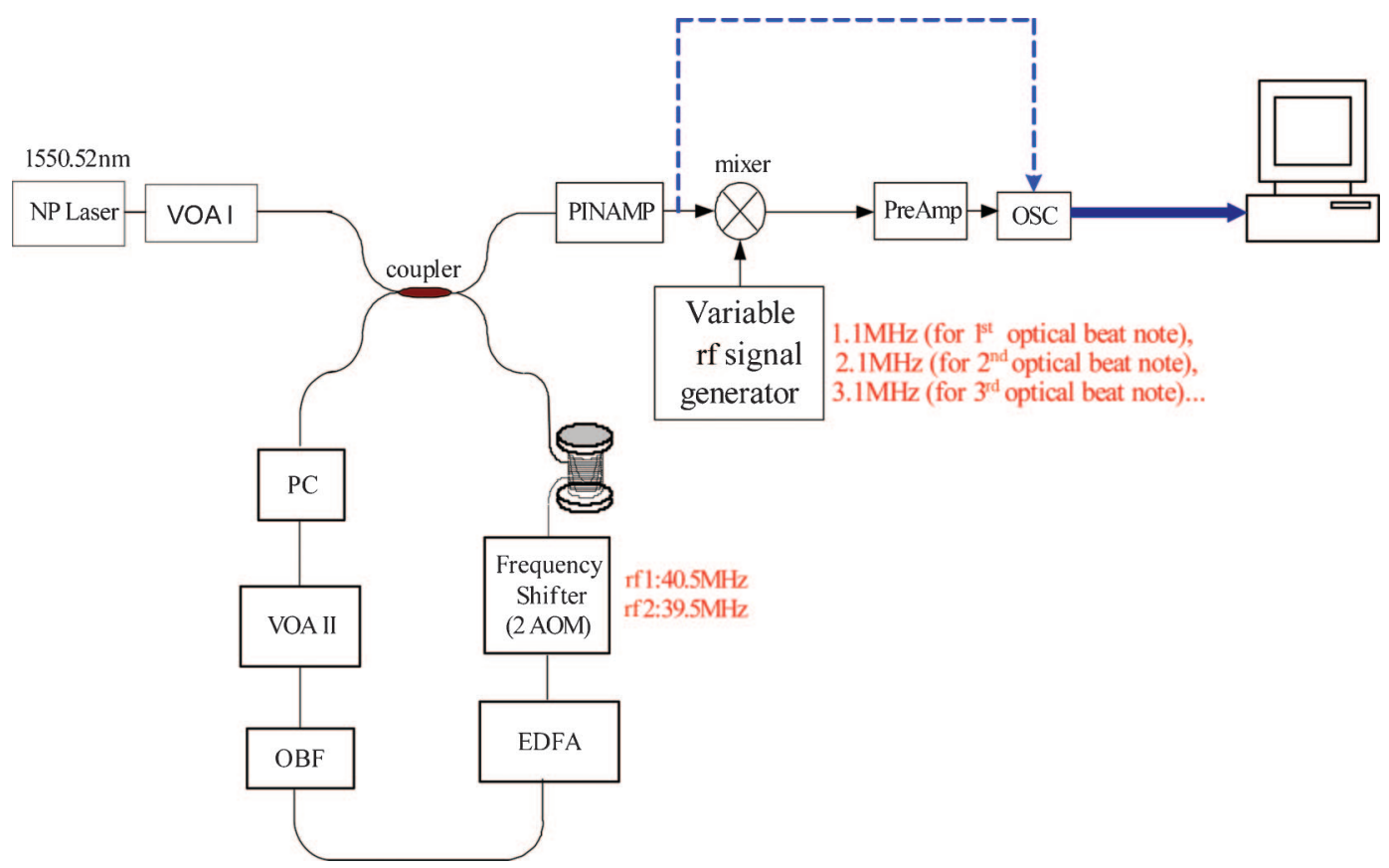

Fig. 1. (Color online) Schematic of experimental setup. OSC, oscilloscope

first time, implemented LC-RDSHI in a subkilohertz laser linewidth measurement.

\section{Experiment Setup}

The system essentially consists of three parts (Fig. 1). The first part is the fiber delay loop consisting of a 2 $\times 2$ fiber coupler; a $25 \mathrm{~km}$ single-mode fiber (SMF); two acousto-optic modulators (AOM) to introduce the appropriate frequency shift; an erbium-doped fiber amplifier (EDFA) to compensate for the losses from the fiber span, the coupler, and the other components involved in the fiber delay loop; an optical bandpass filter (OBF) to suppress the amplified spontaneous emission (ASE) noise from the EDFA; and a polarization controller (PC) to maintain the beam polarization. A variable optical attenuator (VOA) is placed just before the loop to obtain the appropriate optical power input in the delay loop. The second part is signal detection and acquisition, which consists of a p-i-n photodiode with an amplifier (PINAMP) to detect the optical beat signal and to amplify the photocurrent from the detector. An electronic mixer circuit is added to further translate the beat signal to lower frequencies. The last part is signal processing and interpretation in which the acquired data are analyzed by using an appropriate signalprocessing algorithm to determine the linewidth and the frequency noise of the laser being measured.

It is worth mentioning that the $25 \mathrm{~km}$ fiber is in a wooden box surrounded by soundproof microfibers and foam to reduce environmental vibration caused by temperature and acoustical effects. The temperature fluctuations measured at four points in the box are $3.20 \times 10^{-6}{ }^{\circ} \mathrm{C} / \mathrm{s}, 2.87 \times 10^{-6}{ }^{\circ} \mathrm{C} / \mathrm{s}, 3.30$ $\times 10^{-6}{ }^{\circ} \mathrm{C} / \mathrm{s}$, and $3.27 \times 10^{-6}{ }^{\circ} \mathrm{C} / \mathrm{s}$.

The system parameters, such as the frequency shift $\Omega$ introduced by the combined AOM, the coupling co- efficient $\alpha$ of the coupler, the overall optical gain $\gamma$ of the loop, and the attenuation of the VOA, need to be carefully optimized to obtain the best beat signal. There are four considerations in choosing the frequency shift $\Omega$. First, $\Omega$ should be small enough so that the maximum order of the detected beat notes should be within the bandwidth of the photodetector and its following transimpedance amplifier. The second consideration is that $\Omega$ must be much larger than the linewidth of the laser source being measured so that two neighboring beat notes do not overlap. The output spectrum of each order beat note consists of a central line caused by the frequency noise of the laser and the two sidebands from the relaxation oscillation. Therefore the third consideration in setting the value of $\Omega$ is to avoid coupling between the relaxation oscillation and the actual beat notes. The fourth consideration is the environmental perturbation effect on the AOM, so we use two AOMs connected in series. The first AOM shifts the frequency up and the second AOM shifts the frequency down. The frequency fluctuation due to the environmental vibration can therefore be partially canceled. ${ }^{7}$

Another important component is the coupler. The coupling coefficient directly affects the maximum order of the beat notes we can obtain. For example, if $\alpha$ equals $0.1,90 \%$ of the light goes into the loop and we can observe only fewer than 20 orders of beat notes, as shown in Fig. 2(a). To obtain as many orders of beat notes as possible, we chose to use $\alpha$ equals 0.9 with $10 \%$ of the light going into the loop. As many as 150 orders of beat notes can be obtained as shown in Fig. 2(b), in which the frequency interval between each order of beat note is $1 \mathrm{MHz}$.

The third parameter, overall optical gain of the system $\gamma$, must be properly chosen to remove the 


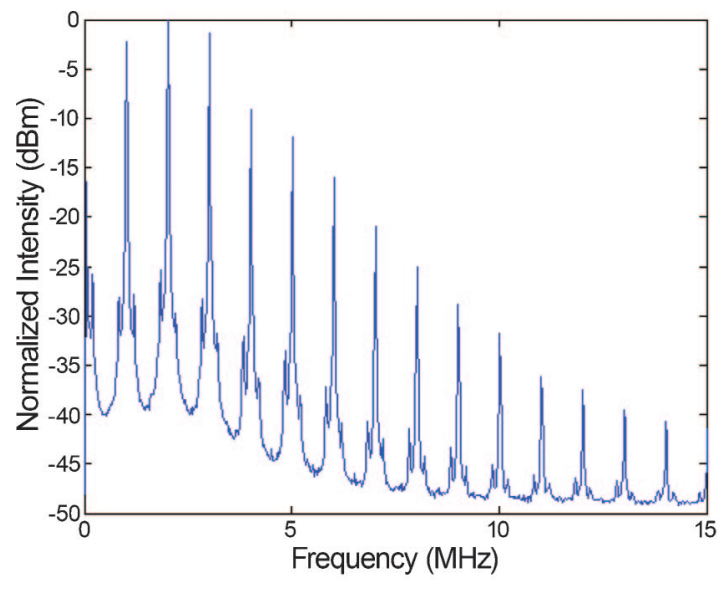

(a)

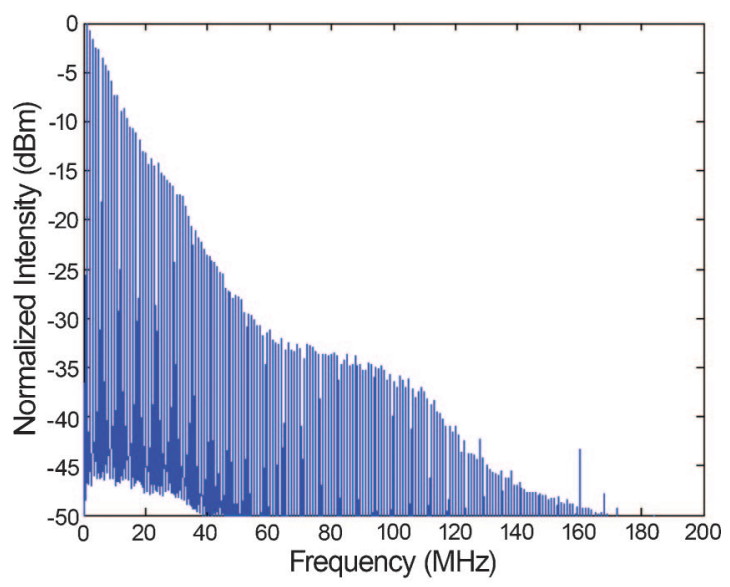

(b)

Fig. 2. (Color online) Effect of coupling coefficient of the coupler (a) $\alpha=0.1$, (b) $\alpha=0.9$.

interferometric effect from multirecirculations of the laser beam in the fiber loop. If we define $S(\omega)$ as the spectrum obtained by DSHI, the spectrum of the $m$ th order of beat note in the LC-RDSHI can be expressed as 6

$$
S_{m}(\omega)=\frac{\gamma^{m}}{(1-\alpha)^{2}} P(\omega) S_{0}\left(\omega, m \tau_{d}, m \Omega\right)
$$

where

$$
P(\omega)=\alpha+\frac{(1-\alpha)\left(\gamma^{2}-\alpha\right)}{1+\gamma^{2}-2 \gamma \cos \left[(\omega+m \Omega) \tau_{d}\right]}
$$

is a periodical function of angular frequency $\omega$, resulting from the multi-interferences of the laser field after multipassing through the loop, and $\tau_{d}$ is the

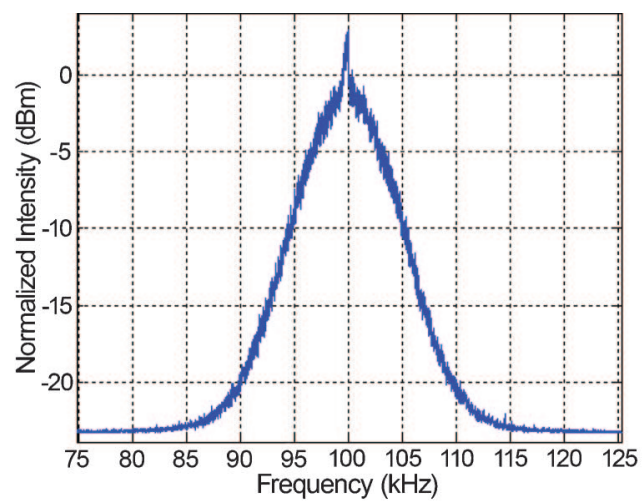

(a)

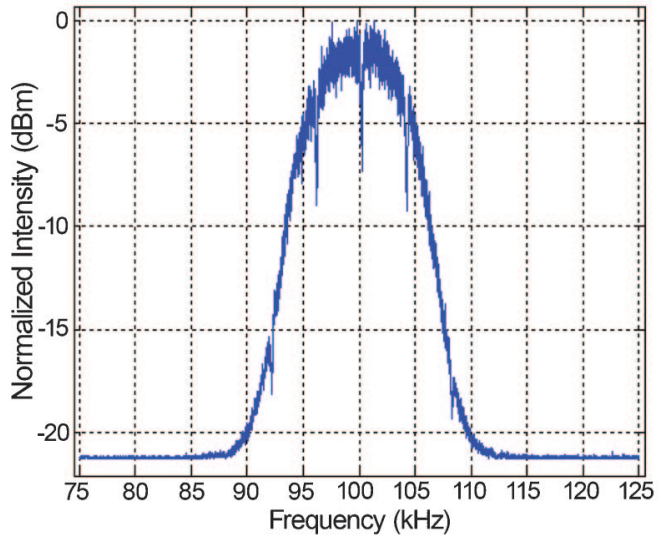

(b)

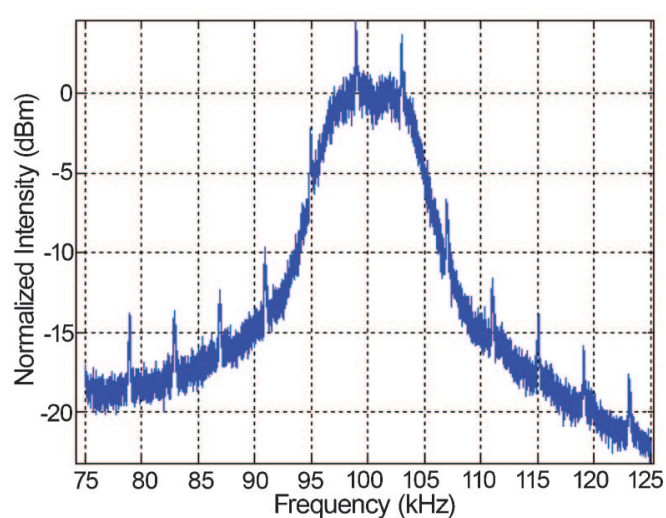

(c)

Fig. 3. (Color online) Tenth-order beat notes with different values of $\gamma$. 
time delay caused by the fiber loop. In Fig. 3, we demonstrate three tenth-order beat notes with different values of $\gamma$. Peaks and notches are observed due to the multirecirculation effect from the LC-RDSHI as predicated by the theoretical analysis in Ref. 6 . To remove the interference effect, the value of $\gamma$ should be either small enough to make the cosine term in Eq. (2) negligible or large enough to make the second term in Eq. (2) approximate a constant. If $\gamma$ happens to be the square root of $\alpha$, the second term in Eq. (2) is zero, and the interference effect can also be removed.

The fourth parameter needed to be set properly is the value of the VOA. If the laser under test has a constant output power, a VOA is needed after the laser to control the input power of the system. The attenuation of the VOA should be set large enough to avoid stimulated Brillouin scattering and small enough to keep the system with a high signal-to-noise ratio.

\section{Experimental Results}

The laser we try to examine is a benchtop erbium microfiber laser module (Scorpion), NP Photonics, Inc. The NP laser is a fiber laser with a cavity established between two fiber Bragg gratings (FBGs). There is a very short piece of active material fusion spliced between the two FBGs. Careful package design removes the vibration or acoustic noise and allows the laser linewidth to be narrower than $1 \mathrm{kHz}$.

Based on the setup shown in Fig. 1, the laser linewidth is measured and the spectrum is interpreted through $20 \mathrm{~dB}$ down direct measurement and Voigt curve fitting.

\section{A. Direct Measurement of $20 \mathrm{~dB}$ Down}

Beat notes centered at $N \times 1 \mathrm{MHz}(\Omega)$ are further transferred to $50 \mathrm{kHz}$ one by one by the mixer circuit. The spectra of the first eight orders of beat notes are shown in Fig. 4. In Fig. 5, we presented linewidth values of these eight orders of beat notes corresponding with their fiber delay lengths. Each linewidth value in Fig. 5 is obtained by taking the bandwidth $20 \mathrm{~dB}$ down from the maximum value of the spectrum shown in Fig. 4 and dividing it by $2 \sqrt{99}$.

The frequency noise spectrum of a laser contains both white frequency noise and $1 / f$ noise. Since $1 / f$ noise will yield a larger measured linewidth, the simplest way to infer a $3 \mathrm{~dB}$ Lorentzian linewidth is to take the linewidth value 10 or $20 \mathrm{~dB}$ down from the spectrum peak. ${ }^{8}$ The Lorentzian linewidth measured by this method is $910 \mathrm{~Hz}$, and the corresponding coherence length is $69.9 \mathrm{~km}$.

\section{B. Voigt Curve Fitting}

In the LC-RDSHI system, the spectrum of each order of beat note is a Voigt profile, which is the convolution of the Lorentzian spectrum associated with the white frequency noise and the approximately Gaussian spectrum arising from the $1 / f$ noise. The second method to extract a Lorentzian linewidth is to use the curve-fitting Voigt profile method. The Voigt spectrum can be expressed as ${ }^{9}$

$$
I(v)=\int_{-\infty}^{\infty} G\left(v^{\prime}\right) L\left(v-v^{\prime}\right) \mathrm{d} v^{\prime} .
$$

$G$ is the normalized Gaussian centered at $v=0$,

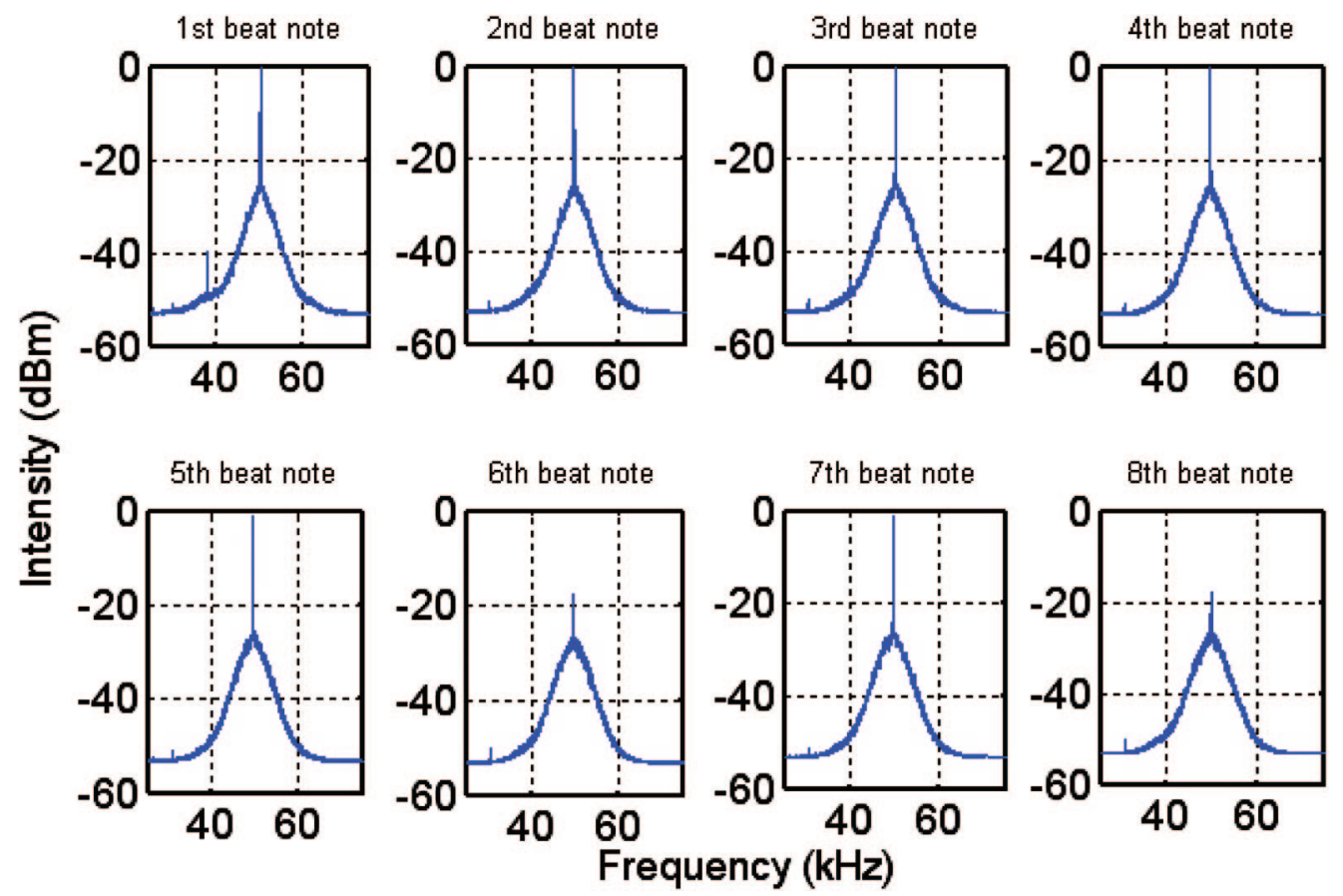

Fig. 4. (Color online) First eight orders of beat notes obtained from the LC-RDSHI. 


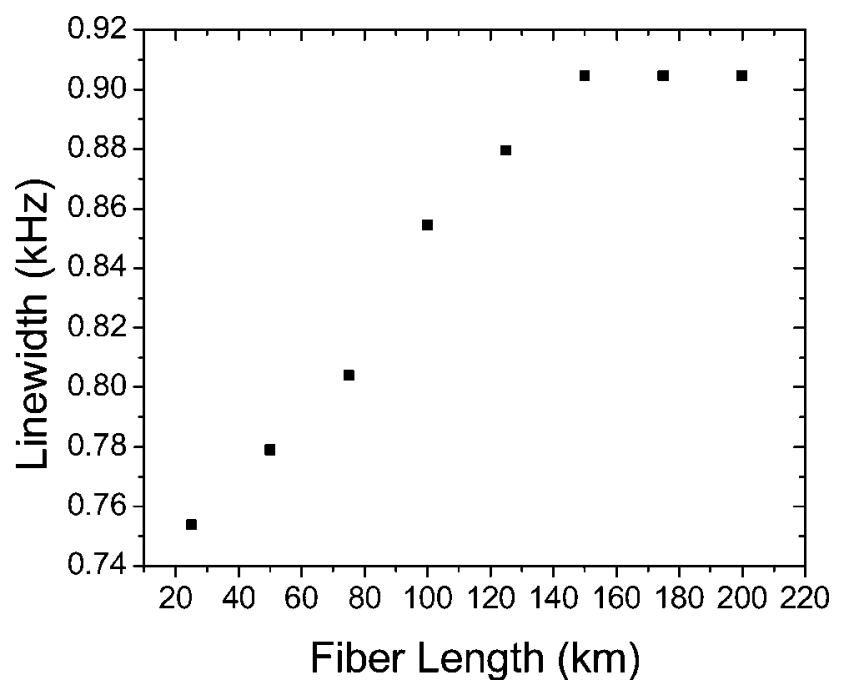

Fig. 5. Laser linewidth from $20 \mathrm{~dB}$ down direct measurement.

$$
G(v)=\frac{\sqrt{4 \ln 2}}{\sqrt{\pi} \Delta v_{\mathrm{GF}}} \exp \left[-(v \sqrt{4 \ln 2})^{2} /\left(\Delta v_{\mathrm{GF}}\right)^{2}\right],
$$

where $\Delta v_{\mathrm{GF}}$ is the FWHM of the Gaussian function. $L$ is the normalized Lorentzian centered at

$$
L(v)=\frac{\Delta v_{\mathrm{LF}}}{2 \pi} \frac{1}{\left(v-v_{0}\right)^{2}+\left(\Delta v_{\mathrm{LF}}\right)^{2} / 4},
$$

where $\Delta v_{\mathrm{LF}}$ is the FWHM of the Lorentzian function.

The Voigt profile function can be manipulated into an expression in terms of the complementary error function of complex argument, $\operatorname{erfc}(z), a^{9,10}$

$$
V(u, a)=\operatorname{Re}\left[\exp \left(z^{2}\right) \operatorname{erfc}(z)\right], \quad z=a+i u,
$$

where

$$
\begin{aligned}
& a=\sqrt{\ln 2} \Delta v_{\mathrm{LF}} / \Delta v_{\mathrm{GF}}, \\
& u=2 \sqrt{\ln 2}\left(v-v_{0}\right) / \Delta v_{\mathrm{GF}} .
\end{aligned}
$$

In Table 1, the Lorenzian linewidth and Gaussian linewidth are obtained by the Voigt curve fitting the

Table 1. Lorentzian Linewidth and Gaussian Linewidth Obtained from Voigt Curve Fitting

\begin{tabular}{ccc}
\hline $\begin{array}{c}\text { Order of Beat } \\
\text { Note }\end{array}$ & $\begin{array}{c}\Delta v_{\mathrm{LF}} \\
(\mathrm{kHz})\end{array}$ & $\begin{array}{c}\Delta v_{\mathrm{GF}} \\
(\mathrm{kHz})\end{array}$ \\
\hline 1st & 0.75 & 4.6 \\
2nd & 0.75 & 4.5 \\
3rd & 0.74 & 4.7 \\
4th & 0.68 & 4.8 \\
5 th & 0.68 & 4.8 \\
6th & 0.68 & 5.1 \\
7th & 0.68 & 5.1 \\
8th & 0.68 & 5.1 \\
\hline
\end{tabular}

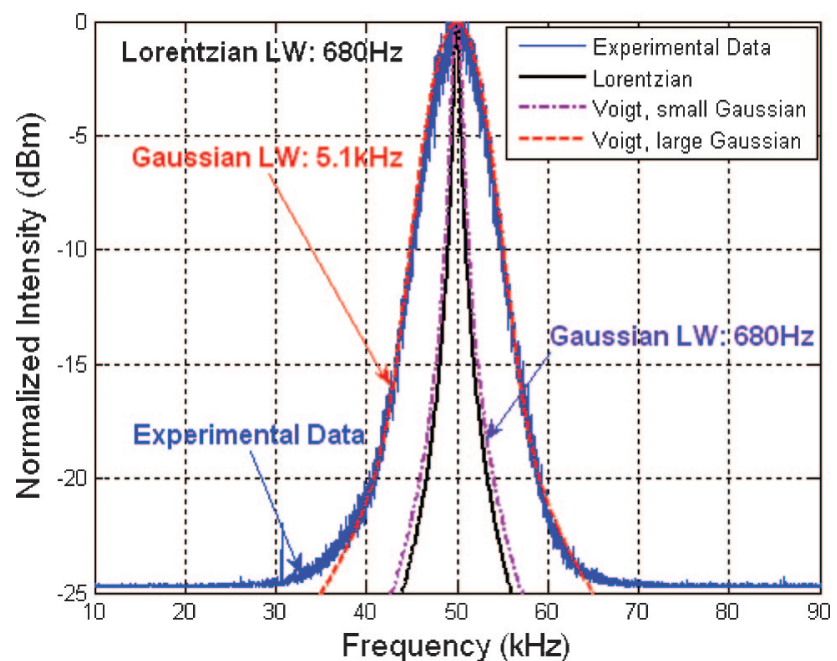

Fig. 6. (Color online) Voigt profile with different Lorenztian and Gaussian linewidths (LWs).

spectra shown in Fig. 4. It is clearly shown that the noise spectrum of the laser under test is the white noise dominant. The Lorenzian linewidth is $680 \mathrm{~Hz}$ from this method. The larger linewidth of the first three orders of beat notes is caused by relaxation oscillation.

Why $20 \mathrm{~dB}$ direct measurement and the Voigt curve-fitting method give different Lorenzian linewidth values can be explained through Fig. 6. As shown in Fig. 6, when the Lorentzian linewidth is $680 \mathrm{~Hz}$, if the laser noise spectrum contains a greater Gaussian component $(5.1 \mathrm{kHz})$, the $20 \mathrm{~dB}$ down direct measurement infers a much larger value of linewidth. If the Gaussian linewidth is smaller than $680 \mathrm{~Hz}$, the $20 \mathrm{~dB}$ down direct measurement provides a simple way to infer the Lorentzian linewidth.

\section{Conclusion}

In this paper the LC-RDSHI was used to measure the ultranarrow laser linewidth. The system was optimized to avoid environmental perturbations and the system parameters were carefully chosen to avoid multirecirculating interference predicted by the theory in Ref. 6.

We can conclude from the Voigt curve fitting that a Gaussian linewidth caused by $1 / f$ frequency noise and other environmental perturbations could be a dominant component in the Voigt profile for ultranarrow linewidth lasers. Therefore the Lorentzian linewidth of an ultranarrow linewidth laser cannot be simply inferred from the $20 \mathrm{~dB}$ down direct measurement.

It is proved that the LC-RDSHI is a unique method for ultranarrow laser linewidth measurement with the self-calibration function. The LC-RDSHI was first developed to improve the resolution of laser linewidth measurement. ${ }^{4,5}$ But it is shown from our measurement results that the LC-RDSHI can also be used for self-calibration. From Fig. 4, we can see that the spectrum of each order of beat note is different. The line- 
width values obtained from these spectra can then be compared with each other and calibrated by the other ones. Every Lorentzian linewidth exacted from the spectrum of each order of beat notes is $680 \mathrm{~Hz}$.

The measurement results also prove that the LCRDSHI is a more flexible method to examine the $1 / f$ noise effect on laser linewidth measurement and the transmission properties of an ultranarrow linewidth laser beam. When we used the Voigt curve-fitting method to interpret the spectrum, it was found that the Lorentzian linewidth is independent of the fiber delay. At the same time, the Gaussian linewidth is highly fiber delay dependent as shown in Table 1.

\section{References}

1. J. Geng, C. Spiegelberg, Y. Hu, Y. Kaneda, S. Jiang, and N. Peyghambarian, "Low noise narrow linewidth fiber laser at 1550 nm," J. Lightwave Technol. 22, 57-62 (2004).

2. S. Jiang, "Single frequency fiber lasers for defense, security and military applications," White Papers, http://www. npphotonics.com.
3. D. Derickson, Fiber Optic Test and Measurement (PrenticeHall, 1998).

4. J. W. Dawson, N. Park, and K. J. Vahala, "An improved delayed self-heterodyne interferometer for linewidth measurement," IEEE Photon. Technol. Lett. 4, 1063-1066 (1992).

5. N. Park, J. W. Dawson, and K. J. Vahala, "Linewidth and frequency jitter measurement of an erbium-doped fiber ring laser by using a loss-compensated, delayed self-heterodyne interferometer," Opt. Lett. 17, 1274-1276 (1992).

6. M. Han and A. Wang, "Analysis of a loss-compensated recirculating delayed self-heterodyne interferometer for laser linewidth measurement," Appl. Phys. B 81, 53-58 (2005).

7. B. K. A. Ngoi and K. Venkatakrishnan, "Techniques to eliminate error induced due to acousto-optic modulator vibration in heterodyne interferometry," Opt. Eng. 38, 2050-2054 (1999).

8. L. B. Mercer, " $1 / f$ frequency noise effects on self-heterodyne linewidth measurements," J. Lightwave Technol. 9, 485-493 (1991).

9. W. J. Thompson, "Numerous neat algorithms for the Voigt profile function," Comput. Phys. 7, 627-631 (1993).

10. J. A. C. Weideman, "Computation of the complex error function," SIAM (Soc. Ind. Appl. Math.) J. Numer. Anal. 31, 1497-1518 (1994). 